\title{
LARGE AMPLITUDE OSCILLATIONS OF A TUBE OF INCOMPRESSIBLE ELASTIC MATERIAL*
}

\author{
BY \\ JAMES K. KNOWLES \\ California Institute of Technology
}

1. Introduction. In recent years a number of problems have been solved in the nonlinear theory of elasticity for incompressible bodies (see [1] and [2] for discussion and references). The work of Rivlin and others has shown that the assumption of incompressibility makes possible the solution of these problems without any special assumptions as to the form of the strain energy function characteristic of the material. Because of the severe non-linearity involved, the special problems considered have been static ones and the methods of attack have been essentially inverse. The question of waves in incompressible materials has been considered by Ericksen [7].

The present paper treats the dynamic problem of axially symmetric oscillations of an infinitely long circular cylindrical tube of incompressible elastic material. The symmetry of the motion and the condition of incompressibility combine to permit the reduction of the problem to one to which the methods of the theory of non-linear vibrations of single-degree-of-freedom systems can be applied. The approach is semi-inverse in the sense that the determination of all physical quantities is made to depend on one unknown function which satisfies a non-linear ordinary differential equation.

The specific problem considered here is that of arbitrary amplitude free oscillations of the tube when it is set in motion under given initial conditions and subject to no surface pressures. It is shown that periodic motions are possible for a large class of strain energy functions, and an expression for the period of oscillation is given in terms of the strain energy function. The results are shown to take a particularly simple form in the limiting case of a thin tube. Explicit results are given for the strain energy corresponding to a material of Mooney-Rivlin type.

The static problem of the symmetrical deformation of a tube of incompressible material by uniform internal and external pressures is a special case of a more general problem solved by Rivlin [3] and discussed in [1]. It may be remarked that results similar to those contained in this paper can be obtained for the radial oscillations of a spherical shell of incompressible material. The corresponding static problem of the symmetrical expansion of a spherical shell under pressure was solved by Green and Shield [4] and is also discussed in [1].

2. Formulation of the problem. Consider an infinitely long circular cylindrical tube of homogeneous, isotropic, elastic, incompressible material which in its undeformed state has inner radius $r_{1}$ and outer radius $r_{2}$. A point in the tube which at time $t$ has cylindrical coordinates $R, \theta, z$ is assumed to have been at the point $r, \theta, z$ in the undeformed state. The motion is thus completely described by the function $R=R(r, t)$.

*Received March 6, 1959. 
If $R_{1}(t), R_{2}(t)$ denote respectively the inner and outer radii at time $t$, the incompressibility condition (see [1]) may be written

$$
R^{2}-R_{1}^{2}=r^{2}-r_{1}^{2}, \quad t \geq 0, \quad r_{1} \leq r \leq r_{2} .
$$

The motion is completely determined if $R_{1}(t)$ is known.

Since the formulation of the present problem differs from that of the corresponding static problem only by the inclusion of the inertia terms in the differential equations of motion, the statement of the complete system of equations of incompressible elasticity and its detailed reduction are omitted and the reader is referred to [1], pp. 88-92. The notation used in this section will be similar to that of [1].

Let

$$
Q=r / R
$$

and temporarily regard $Q$ and $t$ as independent variables.

For an incompressible material the strain energy $W$ per unit undeformed volume is a function of two of the three principal strain invariants $I_{1}, I_{2}, I_{3}: W=W\left(I_{1}, I_{2}\right)$. For the symmetrical deformation $(R Q, \theta, z) \rightarrow(R, \theta, z)$ considered here it is found that

$$
I_{1}=I_{2}=1+Q^{2}+1 / Q^{2} .
$$

Incompressibility requires that $I_{3}=1$, a relation which can be shown to be equivalent to $(2.1)$.

When the stress tensor is calculated in terms of $W$ and the arbitrary hydrostatic pressure $p$, it is found that all shear stresses vanish identically. When the remaining stresses are introduced into the radial equation of motion, there follows the differential equation

$$
\begin{aligned}
\frac{\partial}{\partial Q}\left[p+2 Q^{2} \frac{\partial W}{\partial I_{1}}+2\left(1+Q^{2}\right) \frac{\partial W}{\partial I_{2}}\right]-\frac{2}{Q}\left(1+Q^{2}\right)\left(\frac{\partial W}{\partial I_{1}}+\frac{\partial W}{\partial I_{2}}\right) & \\
& =\frac{R Q}{1-Q^{2}} \rho \frac{\partial^{2} R}{\partial t^{2}},
\end{aligned}
$$

where $\rho$ is the density. The boundary conditions at the inner and outer surfaces take for form

$$
Q=\frac{r_{k}}{R_{k}}: \quad p+2 Q^{2} \frac{\partial W}{\partial I_{1}}+2\left(1+Q^{2}\right) \frac{\partial W}{\partial I_{2}}=-P_{k}(t), \quad k=1,2 .
$$

Here $P_{1}(t)$ and $P_{2}(t)$ are the inner and outer applied pressures, respectively.*

The incompressibility condition (2.1) is now used to compute the acceleration $\partial^{2} R / \partial t^{2}$ in terms of the acceleration $d^{2} R_{1} / d t^{2}$ of particles on the inner surface. Thus

$$
\frac{R Q}{1-Q^{2}} \frac{\partial^{2} R}{\partial t^{2}}=\frac{Q}{1-Q^{2}} R_{1} \frac{d^{2} R_{1}}{d t^{2}}+\left[\frac{Q}{1-Q^{2}}-Q \frac{R_{1}^{2}}{R_{1}^{2}-r_{1}^{2}}\right]\left(\frac{d R_{1}}{d t}\right)^{2} .
$$

Equation (2.6) is now introduced into the right hand side of (2.4), the resulting equation

*The boundary conditions at the "ends" $z= \pm \infty$ of the shell are taken to be of the form of vanishing radial and circumferential shearing stresses and vanishing displacement in the axial direction, so that axially symmetric motion which is independent of $z$ is possible. This of course requires the presence of a suitable axial stress component on the ends of the cylinder. 
is integrated with respect to $Q$ and the boundary condition (2.5) at $Q_{1}=r_{1} / R_{1}$ is imposed. It is found in this way that the hydrostatic pressure $p$ must satisfy

$$
\begin{aligned}
p+2 Q^{2} \frac{\partial W}{\partial I_{1}}+2\left(1+Q^{2}\right) & \frac{\partial W}{\partial I_{2}}=2 \int_{r_{1} / R_{1}}^{Q} \frac{1+Q^{2}}{Q}\left(\frac{\partial W}{\partial I_{1}}+\frac{\partial W}{\partial I_{2}}\right) d Q \\
& +\rho R_{1} \frac{d^{2} R_{1}}{d t^{2}} \int_{r_{1} / R_{1}}^{\Theta} \frac{Q d Q}{1-Q^{2}} \\
& +\rho\left(\frac{d R_{1}}{d t}\right)^{2} \int_{r_{1} / R_{1}}^{0}\left(\frac{Q}{1-Q^{2}}-Q \frac{R_{1}^{2}}{R_{1}^{2}-r_{1}^{2}}\right) d Q-P_{1}(t)
\end{aligned}
$$

The remaining boundary condition then provides the differential equation for $R_{1}(t)$ Thus when (2.5) is imposed at $Q_{2}=r_{2} / R_{2}$ and the integrations in (2.7) are carried out, the following differential equation results.

$$
\begin{gathered}
\rho\left[\frac{1}{2} R_{1} \log \left(\frac{r_{2}^{2}-r_{1}^{2}+R_{1}^{2}}{R_{1}^{2}}\right)\right] \frac{d^{2} R_{1}}{d t^{2}}+\frac{1}{2} \rho\left[\log \left(\frac{r_{2}^{2}-r_{1}^{2}+R_{1}^{2}}{R_{1}^{2}}\right)-\frac{r_{2}^{2}-r_{1}^{2}}{r_{2}^{2}-r_{1}^{2}+R_{1}^{2}}\right]\left(\frac{d R_{1}}{d t}\right)^{2} \\
+2 \int_{r_{1} / R_{1}}^{r_{3}\left(r_{2}^{2}-r_{1}^{2}+R_{1}^{2}\right)-1 / 2} \frac{1+Q^{2}}{Q}\left(\frac{\partial W}{\partial I_{1}}+\frac{\partial W}{\partial I_{2}}\right) d Q=P_{1}(t)-P_{2}(t) .
\end{gathered}
$$

In (2.8) the incompressibility condition has been used to eliminate $R_{2}=\left(r_{2}{ }^{2}-r_{1}{ }^{2}+R_{1}{ }^{2}\right)^{1 / 2}$. The inner radius $R_{1}$ and the velocity $d R_{1} / d t$ are supposed given at time $t=0$.

3. The basic differential equation. The differential equation (2.8) is now rewritten in terms of the following quantities:

$$
\begin{aligned}
& \left.\begin{array}{rl}
x(t) & =R_{1}(t) / r_{1} \\
\mu & =\left(r_{2}^{2} / r_{1}^{2}\right)-1
\end{array}\right\}, \\
& f(x, \mu)=\frac{4}{r_{1}^{2} \rho} \int_{1 / x}^{(\mu+1)^{1 / 2}\left(\mu+x^{2}\right)^{-1 / 2}} \frac{1+Q^{2}}{Q}\left[\left(\frac{\partial W}{\partial I_{1}}+\frac{\partial W}{\partial I_{2}}\right)_{I_{1}=I_{2}=1+0^{2}+1 / Q^{2}}\right] d Q .
\end{aligned}
$$

In this notation (2.8) becomes a non-linear second order differential equation for the dimensionless inner radius $x(t)$ :

$$
x \log \left(1+\frac{\mu}{x^{2}}\right) \frac{d^{2} x}{d t^{2}}+\left[\log \left(1+\frac{\mu}{x^{2}}\right)-\frac{\mu}{\mu+x^{2}}\right]\left(\frac{d x}{d t}\right)^{2}+f(x, \mu)=\frac{P_{1}(t)-P_{2}(t)}{r_{1}^{2} \rho / 2} .
$$

In the sequel (3.3) will be considered only in the case of free oscillations [i.e., $P_{1}(t)=$ $\left.P_{2}(t) \equiv 0\right]$ and subject to the initial conditions

$$
\begin{aligned}
x(0) & =x_{0}, \\
\frac{d x}{d t}(0) & =v_{0} .
\end{aligned}
$$

The function $f(x, \mu)$ which is defined in (3.2) and which appears in the differential equation (3.3) can be rewritten as follows. Let

$$
W_{0}(u)=\frac{2}{r_{1}^{2} \rho} W\left(I_{1}, I_{2}\right)_{I_{1}-I_{2}-1+u+1 / u} .
$$


Then

$$
\frac{d W_{0}}{d u}=\frac{2}{r_{1}^{2} \rho}\left(\frac{\partial W}{\partial I_{1}} \frac{d I_{1}}{d u}+\frac{\partial W}{\partial I_{2}} \frac{d I_{2}}{d u}\right)=\frac{2}{r_{1}^{2} \rho}\left(1-\frac{1}{u^{2}}\right)\left(\frac{\partial W}{\partial I_{1}}+\frac{\partial W}{\partial I_{2}}\right) .
$$

If this fact is used in conjunction with the change of variables $Q=u^{-1 / 2}$ in the integral (3.2), there follows

$$
f(x, \mu)=\int_{\left(\mu+x^{2}\right) /(\mu+1)}^{x^{3}}(u-1)^{-1} \frac{d W_{0}}{d u} d u .
$$

4. Free oscillations. With the notation $v=d x / d t, d^{2} x / d t^{2}=v d v / d x$, it is possible to write the differential equation (3.3) in the form

$$
\frac{d}{d x}\left[\frac{1}{2} v^{2} x^{2} \log \left(1+\mu / x^{2}\right)\right]+x f(x, \mu)=0
$$

and thus to obtain the first integral

$$
\frac{1}{2} v^{2} x^{2} \log \left(1+\mu / x^{2}\right)+F(x, \mu)=C .
$$

The constant $C=\frac{1}{2} v_{0}^{2} x_{0}^{2} \log \left(1+\mu / x_{0}^{2}\right)+F\left(x_{0}, \mu\right)$ depends on the initial values $x_{0}$ and $v_{0}$, and the function $F(x, \mu)$ is given by

$$
F(x, \mu)=\int_{1}^{x} \xi f(\xi, \mu) d \xi=\int_{1}^{x} \xi \int_{\left(\mu+\xi^{2}\right) /(\mu+1)}^{\xi^{2}}(u-1) \frac{d W_{0}}{d u} d u d \xi .
$$

If the order of integration in the repeated integral in (4.3) is reversed, it is possible to carry out one of the integrations and obtain

$$
F(x, \mu)=\frac{1}{2}\left(x^{2}-1\right) \int_{\left(\mu+x^{2}\right) /(\mu+1)}^{x^{2}}(u-1)^{-2} W_{0}(u) d u,
$$

a form which will be convenient later. In obtaining (4.4) it has been assumed that the strain energy vanishes in the undeformed state, so that

$$
W_{0}(1)=0 .
$$

It is well known from the theory of vibrations (see, for example, [5]) that the motion $x(t)$ is periodic if and only if the "energy curves" (4.2) are closed curves in the $x-v$ plane with a finite period $\mathscr{\Phi} d x / v$. We proceed to consider the circumstances under which periodic motions are possible.

It is now assumed* that

$$
\left(\frac{\partial W}{\partial I_{1}}+\frac{\partial W}{\partial I_{2}}\right)_{I_{1}=I_{2}} \geq 0
$$

so that according to (3.6) $d W_{0} / d u$ has the sign of $u-1$ for $u>0$. From this and (3.7) and the fact that $\mu>0$, it follows that

$$
f(x, \mu)\left\{\begin{array}{lll}
<0 & \text { if } & 0<x<1 \\
=0 & \text { if } & x=1 \\
>0 & \text { if } & x>1
\end{array} .\right.
$$

*This restriction on $W$ is implied by inequalities proposed by Truesdell [2], p. 182, and is identical with an inequality obtained by Rivlin and Saunders [6]. 
Consequently the function $F(x, \mu)$ defined in (4.3) decreases monotonically in $x$ for $0<x<1$, vanishes at $x=1$ and increases monotonically for $x>1$.

For real motions $x(t)$ it is always true that $F(x, \mu) \leq C$, so that for every $x$ such that $F(x, \mu)<C$, Eq. (4.2) determines two values of the velocity $v$ :

$$
v= \pm\left(\frac{2 C-2 F(x, \mu)}{x^{2} \log \left(1+\frac{\mu}{x^{2}}\right)}\right)^{1 / 2} \text {. }
$$

To show that the energy curves (4.2) are closed, it is only necessary to show that there are exactly two values of $x$ such that $v=0$ in (4.2); i.e., such that

$$
F(x, \mu)=C .
$$

Since $F(x, \mu)$ decreases monotonically in $x$ for $0<x<1$, there will be exactly one positive root $x<1$ of (4.9) for every positive $C$ unless $\lim x \rightarrow 0+F(x, \mu)$ is finite. If this limit is finite, values of $C$ which exceed it will fail to determine a root $x<1$ of (4.9). Similarly since $F(x, \mu)$ is monotone increasing for $x>1$, there is precisely one root $x>1$ of (4.9) unless $\lim x \rightarrow \infty F(x, \mu)$ is finite. If either of these two limits is finite periodic motions can exist only for sufficiently small $C$.

The conditions that guarantee that the energy curves be closed (and in fact that the motion be periodic) are thus that the integral (4.3) defining $F(x, \mu)$ be unbounded as $x \rightarrow 0+$ and as $x \rightarrow \infty$. To translate these conditions into requirements on the strain energy $W_{0}$, it is convenient to consider $F$ given in the form (4.4). From (4.4) it can be shown directly that $\lim x \rightarrow \infty F(x, \mu)$ will be infinite if only $W_{0}(u)$ is unbounded as $u \rightarrow \infty$. For the case $\lim x \rightarrow 0+F(x, \mu)$ it is observed that by the definition (3.5),

$$
W_{0}(1 / u)=W_{0}(u) .
$$

If the change of variables $u \rightarrow 1 / u$ is then made in (4.4), it is found that

$$
F(x, \mu)=\frac{1-x^{2}}{2} \int_{(\mu+1) /\left(\mu+x^{2}\right)}^{1 / x^{2}}(u-1)^{-2} W_{0}(u) d u .
$$

From this it follows that $\lim x \rightarrow 0+F(x, \mu)=\infty$ if the integral

$$
\int_{1+1 / \mu}^{\infty}(u-1)^{-2} W_{0}(u) d u
$$

diverges. This will be the case if $W_{0}(u)$ increases at least as fast as $u$ as $u \rightarrow \infty$. Hence the energy curves in general will be closed if the strain energy is such that $W_{0}(u)$ becomes infinite at least as fast as $u$ as $u \rightarrow \infty$.

The two roots $x=a<1$ and $x=b>1$ of Eq. (4.9) are the minimum and maximum amplitudes, respectively, of the oscillating inner surface of the cylinder.

The period of the motion is given by

$$
T=2 \int_{a}^{b} \frac{d x}{v}=2 \int_{a}^{b}\left[\frac{x^{2} \log \left(1+\mu / x^{2}\right)}{2 C-2 F(x, \mu)}\right]^{1 / 2} d x .
$$

Since $C-F(x, \mu)$ can vanish only to the first order at $x=a$ and $x=b$, and since $x^{2} \log$ $\left(1+\mu / x^{2}\right)$ is bounded, $T$ is finite.

5. The thin shell. A limiting case which is of interest is that for which $\mu \rightarrow 0$. 
Since $\mu=\left(r_{2} / r_{1}\right)^{2}-1$, the case of small $\mu$ corresponds to the thin shell. The question specifically considered here is that of the limiting form of the maximum and minimum amplitudes of oscillation and of the period as $\mu \rightarrow 0$.

Equation (4.9), which determines the amplitudes $x=a(\mu)<1$ and $x=b(\mu)>1$ may be rewritten as follows when $C$ is expressed in terms of the initial values and after division of both sides by $\mu$.

$$
\frac{F(x, \mu)}{\mu}=\frac{1}{2} x_{0}^{2} v_{0}^{2} \frac{\log \left(1+\mu / x_{n}^{2}\right)}{\mu}+\frac{F\left(x_{0}, \mu\right)}{\mu} .
$$

From the representation (4.4) for $F(x, \mu)$ it is easily shown that for every finite positive $x$

$$
\lim _{\mu \rightarrow 0+} \frac{F(x, \mu)}{\mu}=\frac{1}{2} W_{0}\left(x^{2}\right) .
$$

Also

$$
\lim _{\mu \rightarrow 0+} \frac{1}{2} x_{0}^{2} v_{0}^{2} \frac{\log \left(1+\mu / x_{0}^{2}\right)}{\mu}=\frac{1}{2} v_{0}^{2} .
$$

If $a_{0}$ and $b_{0}$ denote the limiting values as $\mu \rightarrow 0$ of the amplitudes $a(\mu)$ and $b(\mu), a_{0}$ and $b_{0}$ satisfy the limiting form of (5.1) as $\mu \rightarrow 0$.

$$
W_{0}\left(x^{2}\right)=v_{0}^{2}+W_{0}\left(x_{0}^{2}\right) .
$$

From the property $W_{0}(1 / u)=W_{0}(u)$ it follows that the maximum and minimum limiting amplitudes $a_{0}$ and $b_{0}$ are related by

$$
b_{0}=\frac{1}{a_{0}} .
$$

Now consider the expression (4.11) for period $T$. In the limit $\mu \rightarrow 0$ the results (5.2), (5.3) and (5.5) show that

$$
T_{0}=\lim _{\mu \rightarrow 0+} T=2 \int_{a_{0}}^{1 / a_{0}}\left[v_{0}^{2}+W_{0}\left(x_{0}^{2}\right)-W_{0}\left(x^{2}\right)\right]^{-1 / 2} d x
$$

is the limiting value of the period of the motion as $\mu \rightarrow 0$.

It may be remarked that while the present discussion has obtained only the limiting values of the amplitudes and the period as $\mu \rightarrow 0$, it is possible by further calculation to obtain the correction terms of order $\mu$, say, in the formal expansions of these quantities for small $\mu$.

6. The Mooney-Rivlin material. It is interesting to examine the results of Sec.4 and 5 for the case of the Mooney-Rivlin material (see [1], p. 76) for which the strain energy function is given by

$$
W\left(I_{1}, I_{2}\right)=\frac{1}{2} \alpha\left(I_{1}-3\right)+\frac{1}{2} \beta\left(I_{2}-3\right),
$$

where $\alpha$ and $\beta$ are positive constants.

According to (3.5) the function $W_{0}(u)$ assumes the form

$$
W_{0}(u)=K\left(u+\frac{1}{u}-2\right) \text {, }
$$

where

$$
K=(\alpha+\beta) / r_{1}^{2} \rho .
$$

When the integrations in (4.4) are carried out it is found that 


$$
F(x, \mu)=\frac{1}{2} K\left(1-x^{2}\right) \log \left(\frac{1+\mu / x^{2}}{1+\mu}\right) .
$$

For this special case $F(x, \mu)$ is unbounded as $x \rightarrow 0$ and as $x \rightarrow \infty$, so that periodic motions exist for all values of the initial conditions.

In the limiting case of the thin shell the condition (5.4) for the amplitudes becomes

$$
x^{2}+\frac{1}{x^{2}}=\frac{v_{0}^{2}}{K}+x_{0}^{2}+\frac{1}{x_{0}^{2}} .
$$

The root $a_{0}<1$ of this equation is readily found to be

$$
a_{0}=\left[\frac{v_{0}^{2}}{2 K}+\frac{x_{0}^{2}}{2}+\frac{1}{2 x_{0}^{2}}-\frac{1}{2}\left(\frac{v_{0}^{4}}{K^{2}}+x_{0}^{4}+\frac{1}{x_{0}^{4}}+\frac{2 x_{0}^{2} v_{0}^{2}}{K}+\frac{2 v_{0}^{2}}{K x_{0}^{2}}-2\right)^{1 / 2}\right]^{1 / 2}
$$

while the maximum amplitude is given by $b_{0}=1 / a_{0}$.

For the initial conditions $x_{0}=1, v_{0} \neq 0$, corresponding to the situation in which the tube is set in motion by imparting to it an initial velocity in its undeformed state, Eq. (6.5) becomes

$$
\left.\begin{array}{l}
a_{0}=\left[1+\frac{v_{0}^{2}}{2 K}-\frac{1}{2}\left(\frac{v_{0}^{4}}{K^{2}}+\frac{4 v_{0}^{2}}{K}\right)^{1 / 2}\right]^{1 / 2} \\
b_{0}=\frac{1}{a_{0}}
\end{array}\right\} .
$$

On the other hand for the initial conditions $x_{0} \neq 1, v_{0}=0$, so that the tube is released from rest in an expanded or contracted state, Eq. (6.5) shows that the inner radius of the tube oscillates between its initial value and the reciprocal of that value.

The expression (5.6) for the limiting value of the period takes the form

$$
T_{0}=2 K^{-1 / 2} \int_{a_{0}}^{1 / a_{0}}\left(\frac{v_{0}^{2}}{K}+x_{0}^{2}+\frac{1}{x_{0}^{2}}-x^{2}-\frac{1}{x^{2}}\right)^{-1 / 2} d x
$$

where $a_{0}$ is given by (6.5). When this integral is evaluated it is found that

$$
T_{0}=\pi K^{-1 / 2}=\pi\left(\frac{r_{1}^{2} \rho}{\alpha+\beta}\right)^{1 / 2} \text {. }
$$

Thus in the limit $\mu \rightarrow 0$ the period of oscillation does not depend on the amplitude or any other characteristics of the motion for the Mooney-Rivlin material.

\section{REFERENCES}

1. A. E. Green and W. Zerna, Theoretical elasticity, Clarendon Press, Oxford, 1954

2. C. Truesdell, The mechanical foundations of elasticity and fluid dynamics, J. Ratl. Mech. Anal. 1, $125-300$ (1952)

3. R. S. Rivlin, Large elastic deformation of isotropic materials VI. Further results in the theory of torsion, shear and flexure. Phil. Trans. Roy. Soc. London (A) 242, 173-195 (1949)

4. A. E. Green and R. T. Shield, Finite elastic deformation of incompressible isotropic bodies Proc. Roy. Soc. London (A) 202, 407-419 (1950)

5. J. J. Stoker, Non linear vibrations, Interscience Publishers, New York, 1950

6. R. S. Rivlin and D. W. Saunders, Large elastic deformation of isotropic materials VII. Experiments on the deformation of rubber. Phil. Trans. Roy. Soc. London (A) 243, 251-288 (1951)

7. J. L. Ericksen, On the propagation of waves in isotropic incompressible perfectly elastic materials, J. Ratl. Mech. Anal. 2, 329-337 (1953) 